\title{
Parto humanizado: benefícios e barreiras para sua implementação
}

\author{
Humanized delivery: benefits and barriers to its implementation \\ Parto humanizado: beneficios y barreras para su implementación
}

Recebido: 13/11/2021 | Revisado: 21/11/2021 | Aceito: 22/11/2021 | Publicado: 02/12/2021

\author{
Esther Lima da Silva \\ ORCID: https://orcid.org/0000-0002-2461-706X \\ Universidade Paulista, Brasil \\ E-mail: esther_limaestrela@hotmail.com \\ Maria Eduarda Arnaud de Andrade \\ ORCID: https://orcid.org/0000-0001-7986-8918 \\ Universidade Paulista, Brasil \\ E-mail: mariaeduardandrade28@gmail.com \\ Sarah Stefany de Lima Carvalho \\ ORCID: https://orcid.org/0000-0001-6958-0988 \\ Universidade Paulista, Brasil \\ E-mail: sarahstefanys@gmail.com \\ Valéria Leonhardt \\ ORCID: https://orcid.org/0000-0002-7701-6284 \\ Universidade Paulista, Brasil \\ E-mail: valeria.leonhardt@docente.unip.br \\ Maria Luiza Rêgo Bezerra \\ ORCID: https://orcid.org/0000-0002-3336-7760 \\ Universidade Paulista, Brasil \\ E-mail: maria.bezerra@ docente.unip.br
}

\begin{abstract}
Resumo
O conceito de parto humanizado vai além da ideia de conforto e a minimização da dor no momento do parto, mas abrange um conjunto de medidas desde o pré-natal até o pós-parto, que visam proporcionar à mulher um grau elevado de satisfação, autonomia e segurança. Este estudo é uma revisão bibliográfica, que visa compreender a importância da humanização do parto para o binômio mãe-bebê e identificar as barreiras enfrentadas pelos profissionais de saúde para sua implementação. Para a realização da pesquisa, foram utilizada as bases de dados BVSalud, SCIELO, por meio do descritor controlado "Parto Humanizado", busca livre com os termos: "Pai and parto humanizado" e "Parto e mulheres negras" além de consultas em sites oficiais relacionados para saúde. Para inclusão de artigos, foi filtrados pelos anos de 2016 até 2021 com língua portuguesa, inglesa ou espanhola e textos completos condizentes com o assunto da pesquisa a ser realizada. Os resultados dos estudos apontaram que o processo de humanização contribui para uma melhor vivência do parto e puerpério para a mulher, beneficiando consequentemente o bebê e toda rede familiar. Porém, para o sucesso desse processo, é importante o conhecimento técnico-científico dos profissionais de saúde responsáveis, bem como o suporte para sua implementação. Tais fatores parecem interferir processo de humanização.
\end{abstract}

Palavras-chave: Parto humanizado; Humanização do parto; Enfermagem obstétrica; Programa de humanização no pré-natal e nascimento; Parto normal.

\begin{abstract}
The concept of humanized childbirth goes beyond the idea of comfort and the minimization of pain at the time of delivery, but covers a set of measures from prenatal to postpartum, which aim to provide women with a high degree of satisfaction, autonomy, and safety. This study is a literature review, which aims to understand the importance of the humanization of childbirth for the mother-baby binomial and to identify the barriers faced by health professionals for its implementation. To carry out the research, the databases BVSalud and SCIELO were used, using the controlled descriptor "Humanized Childbirth", free search with the terms: "Father and humanized childbirth" and "Childbirth and black women" in addition to consultations on official websites related to health. For inclusion of articles, it was filtered by the years from 2016 to 2021 with Portuguese, English or Spanish language and full texts consistent with the subject of the research to be performed. The results of the studies pointed out that the humanization process contributes to a better experience of childbirth and puerperium for the woman, consequently benefiting the baby and the entire family network. However, for this process to be successful, the technical and scientific knowledge of the health professionals in charge is important, as well as the support for its implementation. Such factors seem to interfere with the humanization process.
\end{abstract}

Keywords: Humanized childbirth; Humanization of childbirth; Obstetric nursing; Prenatal and birth humanization program; Normal childbirth. 


\begin{abstract}
Resumen
El concepto de parto humanizado va más allá de la idea de comodidad y minimización del dolor en el momento del parto, sino que abarca un conjunto de medidas desde el prenatal hasta el posparto, que tienen como objetivo proporcionar a las mujeres un alto grado de satisfacción, autonomía y seguridad. Este estudio es una revisión bibliográfica, cuyo objetivo es conocer la importancia de la humanización del parto para el binomio madre-bebé e identificar las barreras a las que se enfrentan los profesionales sanitarios para su implantación. Para la realización de la investigación, se utilizaron las bases de datos BVSalud, SCIELO, mediante el descriptor controlado "Parto Humanizado", que busca libros con los términos: "El padre y el parto humanizado" y "El parto y las mujeres negras", además de las consultas en páginas web oficiales relacionadas con la salud. Para la inclusión de los artículos, se filtró por los años de 2016 a 2021 con idioma portugués, inglés o español y textos completos coherentes con el tema de la investigación a realizar. Los resultados de los estudios apuntan a que el proceso de humanización contribuye a una mejor vida del parto y el puerperio para la mujer, beneficiando en consecuencia al bebé y a toda la red familiar. Sin embargo, para el éxito de este proceso, es importante el conocimiento técnico-científico de los profesionales sanitarios responsables, así como el apoyo para su aplicación. Estos factores parecen interferir en el proceso de humanización.

Palabras clave: Parto humanizado; Humanización del parto; Enfermería obstétrica; Programa de humanización en el parto y nacimiento; Parto normal.
\end{abstract}

\title{
1. Introdução
}

A concepção de parto humanizado vai além da ideia de conforto e a minimização da dor no momento do parto, mas, abrange um conjunto de medidas desde o pré-natal até o pós-parto, que objetivam proporcionar a mulher um grau elevado de satisfação, autonomia e segurança. Assim, a gestante deve ser acolhida pelo profissional de enfermagem capacitado, de forma que a proporcione o que for de sua vontade e necessidade, garantindo um parto de forma tranquila e saudável (Moura et al., 2020).

No entanto, a ideia da escolha pelo parto cirúrgico vem de questões distorcidas. Intervenções desnecessárias vêm aumentando e carregando uma visão desvirtuada quanto ao uso das tecnologias. Tal realidade, manifesta sobre o parto uma definição contrária ao que realmente a humanização descreve: uma expressão de saúde. Os fatos citados, diversas vezes são frutos da persuasão médicas de forma suasória, mas deveria ser de livre escolha da gestante. Como forma de benefícios unilaterais, a insistência pela escolha do parto cirúrgico muitas vezes é infeliz para o binômio mãe-bebê (Monteiro et al., 2020).

No Brasil, o apropriado de partos cesáreos é de no máximo 15\%. Porém a realidade nos mostra que por volta de $55,6 \%$ são cirúrgicos. Infelizmente na rede privada os números são piores, chegando a 84,6\%. O ministério de saúde em 2017 revela que foram realizados 2,7 milhões de partos no país. As estatísticas reveladas pelo Sistema único de Saúde (SUS) apresenta-se melhor que da rede privada, porém distante da meta, apresentando $58,1 \%$ de partos normais e $41,9 \%$ de partos cesáreos. Tais números são relevantes, uma vez que $98 \%$ dos nascimentos ocorrem nos hospitais (Monteiro et al., 2020).

É imprescindível no que se refere ao âmbito da enfermagem, inicialmente conhecer, estudar e colocar em pratica todos os princípios do Programa de Humanização no Pré-Natal e Nascimento (PHPN), tais como: o direito de conhecer e acessar a maternidade onde as gestantes serão atendidas no momento de parto, direito a um atendimento de qualidade e digno desde o parto até o puerpério, de uma assistência humanizada, segura e eficaz de acordo com as condições e princípios gerais determinados pelo conhecimento cientifico. O recém-nascido é digno, assim como a mãe, de uma assistência neonatal segura e humanizada (Monteiro et al., 2020).

Diante do exposto, questiona-se: Quais os benefícios do parto humanizado e o que se tem como barreira para sua execução? Este estudo tem a intensão de levantar dados da literatura sobre os benefícios do parto humanizado para a parturiente e o bebê, além das dificuldades enfrentadas pelos profissionais de saúde para a realização do Parto Humanizado.

Assim sendo, espera-se que esta pesquisa possa despertar nos leitores sentimentos humanitários referente a saúde da mulher durante todo período gestacional (desde o pré-natal até o puerpério) e do neonato. 


\section{Metodologia}

Este estudo trata-se de uma revisão bibliográfica de formato narrativa. Para a realização da pesquisa, foi utilizada as bases de dados BVSalud, SCIELO, através de pesquisa livre pelos termos "Parto Humanizado", "Pai and parto humanizado", "Parto e mulheres negras", "Dificuldades and Parto Humanizado" e "políticas de humanização do parto", além de consultas à cartilhas de sites oficiais relacionados à saúde. Para a inclusão dos artigos, filtrou-se pelos anos de 2016 até 2021 com idioma português, inglês ou espanhol e textos completos condizentes com o assunto da pesquisa. Após a seleção dos artigos, foi realizada pelas autoras da pesquisa uma leitura prévia, seguida de leitura minuciosa. Já como método de exclusão, foram eliminados artigos em que seus temas não fizessem relação ao tema do trabalho, que não houvesse textos completos e publicados anterior ao ano de 2016 com exceção dos artigos encontrados pelo termo "políticas de humanização do parto".

A coleta de dados para o desenvolvimento desta pesquisa foi realizada primeiramente pela base de dados BVSalud com o termo "Parto Humanizado", totalizando 456 artigos. Posteriormente realizou-se uma seleção de artigos que se enquadravam nos tópicos a serem abordados no decorrer da pesquisa através da leitura dos resumos, totalizando 13 artigos que seriam utilizados no trabalho. Em seguida, utilizando o termo "Pai and Parto humanizado", foram encontrados 32 artigos, dos quais filtrou-se 8, selecionando apenas 06. Pesquisando também pelo termo "Parto mulheres negras", na plataforma Scielo, encontrou-se 08 artigos, selecionado apenas 02, em contrapartida, na plataforma BVSalud, foram encontrados 27 artigos que também incluíam os encontrados na plataforma Scielo, porém, selecionados apenas 02, totalizando 04 artigos para esse termo. Ainda na plataforma BVSalud, foi pesquisado pelo termo “dificuldades and parto humanizado" da qual encontrou-se 27 artigos e foram selecionados 2 artigos. Para o tópico "Políticas públicas" foi realizada uma pesquisa separada na plataforma BVSalud com o termo "políticas de humanização do parto" incluindo textos de todos os anos, sendo selecionados 04 artigos, 01 cartilha, 01 folder, assim como pesquisa no site do Ministério da Saúde relacionadas à humanização. Assim, a pesquisa teve um total de 27 artigos em todo seu corpo.

Para análise e inclusão dos dados encontrados, utilizou-se como artifícios a leitura minuciosa sendo exploratória, seletiva e de forma a incluir informações associadas ao parto humanizado, consistindo em: seus benefícios, participação do pai como parte da humanização, parto humanizado e mulheres negras, políticas públicas relacionadas à humanização do parto e barreiras para a implementação. Foi realizada também, uma busca livre a sites oficiais como Ministério da Saúde, da qual foram encontradas cartilhas informativas que também contribuíram para o conteúdo do trabalho.

\section{Resultados e Discussão}

Os resultados encontrados guiaram à descrição de temas que são pertinentes para o desenvolvimento completo do assunto, sendo eles: Parto humanizado - benefícios e barreiras; Presença paterna como parte da humanização; Parto humanizado e raça; Políticas públicas; Parto humanizado: Barreiras para sua implementação.

\section{Parto Humanizado: Definições e benefícios}

A definição de maternidade vem se desenvolvendo desde meados do século XX, de forma vasta e bastante variável conforme culturas. Desde então, o conceito de maternidade tem sido compreendido como uma experiência que abrange, de forma prioritariamente emocional, a relação entre o corpo da mulher e o bebê. Assim sendo, o enfoque dessa experiência é, especialmente, tripartite, abrangendo a gravidez, o parto e o puerpério, dando origem ao nominado parto humanizado, a amamentação exclusiva, prolongada e por livre demanda, a criação com apego, etc (Russo \& Nucci, 2020).

O termo de humanização é visto de diferentes formas de acordo com cada profissional de saúde. A visão de alguns profissionais é de que o parto humanizado é sinônimo de parto vaginal. Em contrapartida, outros acreditam que para um maior suporte emocional e até mesmo físico, é necessário a presença do acompanhante. Entretanto, é imprescindível levar em 
consideração a opinião da mulher. Sem este fator, não se pode levar em conta a humanização, visto que os atores principais da cena são ela, o recém-nascido e a família em questão. Desta forma, levando em conta os valores da humanização, é importante citar o empoderamento feminino, as crenças da mulher, assim como seu estado emocional e durante o parto, enaltecendo-a com sua autonomia e dignidade (Cordeiro et al., 2018).

No Brasil, o conceito de Humanização se dá de forma que expresse formas de cuidados direcionados às mulheres durante e após a gestação. Sendo assim, a partir de 2000, inicia-se a fomentação de programas de humanização que acolhem a saúde materna. O posicionamento crítico das autoras ao modelo de obstetrícia dá origem às teses e dissertações que descrevem a humanização segundo um novo modelo (Bourguignon \& Grisotti, 2020).

Em 2002, no Brasil, foi instituído pelo Ministério da Saúde (MS) o programa de Humanização no Pré-Natal e Nascimento (PHPN), a fim de garantir os direitos de escolha da mulher, a reorganização da assistência e o parto com o mínimo de intervenções. Assim sendo, com objetivo da diminuição da mortalidade neonatal também pertinente aos nascimentos cirúrgicos, em 2011 implementou-se a Rede Cegonha (Baggio et al, 2021).

Isto posto, para o processo de humanizar, é imprescindível que se obtenha no atendimento reconhecimento da individualidade, além de estabelecer um vínculo com cada mulher de acordo com a demanda e as necessidades individuais, não havendo prática de relações autoritárias e desiguais. Lidar com a passagem parturitiva, é ser capaz de proporcionar tranquilidade e segurança para o bebê e à puérpera (Leal et al, 2021).

As boas práticas são melhores apresentadas quando assistidas por Enfermeiros Obstétricos, uma vez que, na presença dos mesmos, evidencia-se menores incidências de más práticas. A confirmação desse processo vem através de mulheres que expressam pouco sofrimento e muita satisfação diante da experiência do parto natural. Além de se sentir vitoriosa por conseguir dar à luz, possuem também uma recuperação eficaz e rápida, menos dor após o parto, insignificantes chances de complicações, hemorragias, infecções, além de poder retornar as atividades diárias normalmente em curto prazo e ter alta hospitalar em período mínimo (Baggio et al., 2021).

Para proporcionar conforto à mulher, as tecnologias de cuidado auxiliam no trabalho de parto e nascimento, estimulando também com que a mesma tenha autonomia durante todo processo (Lima et al., 2020). Dentre essas tecnologias, pode-se citar algumas aos quais são efetivas para um maior conforto a mulher sem o uso de métodos farmacológicos. Indicados para alívio da dor, o banho quente, massagens, incentivo para respiração tranquila e exercícios perianais serão efetivos. Já direcionadas a progressão da apresentação fetal, manter a mulher relaxada, calma e concentrada e incentivando a adoção de posições verticais para proporcionar maior dilatação cervical e menor uso de analgesia, tornam-se prática que contribuirão para o estimulo do parto normal e bem-estar da mulher (Baggio et al., 2021).

De acordo com um estudo qualitativo com 12 mulheres, por meio de entrevistas semiestruturadas, após 60 dias do parto, a visão das parturientes que passaram pelo processo do parto normal, é que o parto fisiológico apresenta dor, porém é suportável. Ressaltam também o parto normal como uma experiência satisfatória, ao qual a lembrança dolorosa é reduzida após o parto. Assim sendo, evidencia-se que a recuperação pós-parto humanizado é melhor que a operação cesariana, na qual, no começo, há ausência de dor, porém, após o efeito da anestesia, há a possibilidade de haver uma dor persistente na parturiente. Salienta-se que a puérpera fica limitada em seus movimentos, pode haver problemas na cicatrização e incômodo da sutura de acordo com o tempo (Baggio et al., 2021).

A importância de melhoras as experiências das mulheres durante a gravidez e o parto é salientada pela Organização Mundial da Saúde (OMS). Essa melhoria é alcançada através de uma atenção integralizada e humanitária, bem como da garantia de não sofrer violência ou qualquer tipo de represália, que acarretam em danos morais e psíquicos. (Villamil et al., 2020). 


\section{Presença paterna como parte da humanização}

Tendo em vista que o parto é um processo de grande importância, vivenciado de uma forma tão intensa e de extrema individualidade de cada puérpera, esse momento único deve ser compartilhado com alguém que traga à gestante confiança e segurança, para que se torne um momento agradável e de grande conforto (Holanda et al., 2018).

No ano de 2005 instituiu-se a Lei $\mathrm{n}^{\circ} 11.108$, garantindo a presença de um acompanhante junto à parturiente durante $\mathrm{o}$ trabalho de parto, o parto e o pós-parto nos serviços de saúde do Sistema Único de Saúde, pois, em anos anteriores, a escolha pela presença do pai ou de outro acompanhante na sala de parto, dependia do consenso da instituição ao qual a parturiente se encontrava (Francisco et al., 2015).

O Ministério da Saúde afirma que a parturiente necessita de alguém que torne o trabalho de parto e puerpério um momento de conforto e tranquilidade, trazendo à tona um momento mais seguro e com total colaboração para que o neonato e a parturiente saiam desse processo de modo melhorado e com uma vivencia única e tranquila, além de ser parte dos seus direitos. Então, no Brasil, é recomendado que toda parturiente tenha alguém do seu lado que seja de sua escolha e que lhe traga tranquilidade, coragem e conforto em todo processo (Holanda et al., 2018).

A relevância do papel do pai na promoção da saúde reprodutiva e sexual do homem está explícito na Política Nacional de Atenção Integral à Saúde Do Homem (PNAISH). Nessa mesma direção, a Rede Cegonha valoriza a presença do pai no parto para a humanização do cuidado tendo como estratégia a qualificação da atenção à saúde obstétrica e infantil. Nessa mesma temática, é possível criar várias possibilidades para imaginar e exercer os direitos e cuidados, incluindo no âmbito da sexualidade e paternidade. Tal fator contribui para a construção da imagem masculina, contribuindo também para a redução da vulnerabilidade feminina e masculina (Braide et al., 2018).

Estudos apontam, que a presença do pai no momento do parto, leva a diminuição do desconforto da mulher. A presença paterna se faz de grande importância para uma experiência agradável para a mulher, assim, podendo ser considerada uma das estratégias da humanização do parto, sendo também um método não farmacológico para a redução da dor. Diante disto, consequentemente, pode-se esperar a diminuição de procedimento e intervenções desnecessárias durante o processo de trabalho de parto e parto (Quitete \& Monteiro, 2018).

O pai também se caracteriza por se tornar referência familiar, apoio emocional e suporte nos cuidados a mulher. Sendo assim, se torna peça importante por proporcionar fortalecimento no trabalho de parto, além de mais conforto, segurança e tranquilidade, sendo refletido também no relacionamento conjugal e contribuindo para o empenhamento do pai com a qualidade de vida e saúde da família (Ribeiro et al., 2018).

A relação pai-bebê durante a gestação, tem total relevância na relação pai-filho pós nascimento por se tornar peça fundamental na estruturação da relação familiar, sendo de grande importância incluir esse fato valorizando a paternidade e seus limites na sociedade (Menezes et al., 2019).

Tendo em vista os benefícios da presença do companheiro, é imprescindível o acolhimento e incentivo ao companheiro desde o pré-natal até o parto, minimizando assim os medos e anseios com o nascimento, e compreendendo que a participação paterna durante este processo deve-se iniciar o mais rápido possível (Quitete \& Monteiro, 2018).

\section{Parto humanizado e raça}

Por mais de três séculos, o Brasil vivenciou a escravidão, onde o negro era desvalorizado e discriminado por suas características tanto físicas como culturais, o que evidenciava a dominação dos brancos (Oliveira \& Kubiak, 2019).

No que tange à saúde, atualmente, é efetivado pela Constituição Federal Brasileira princípios do Sistema Único de Saúde (SUS) universal, igualitário e integral, que garantem o direito ao acesso à saúde de forma a respeitar a individualidade de cada indivíduo, assegurando a promoção, proteção e recuperação da saúde (Theophilo et al., 2017). 
Assim sendo, apesar da proibição pela lei, pode-se observar que ainda há diversas formas de racismo, e como exemplo, tem-se o racismo praticado pelas instituições em suas formas de tratarem tais indivíduos de forma a desrespeitarem os seus direitos (Oliveira \& Kubiak, 2019).

No âmbito da prestação de serviços, o racismo se caracteriza pela restrição ao acesso à saúde da população negra, bem como a relação entre os profissionais de saúde. Na assistência à saúde, pode-se identificar situações as quais os profissionais criam um sistema de ideias distorcidas que desrespeitam a individualidade do sujeito como "negros são mais resistentes a dor" ou "negros não adoecem". Tais ideias acabam a reduzir a qualidade da assistência igualitária e integral, destacando o declínio no cuidado de mulheres negras nos procedimento de pré-natal e parto (Oliveira \& Kubiak, 2019).

Estima-se que no Brasil, 1 em cada 4 mulheres sofrem algum tipo de violência no parto (Lima \& Moreira \& Pimentel, 2016). Mulheres negras por sua vez se tornam maioria tratando-se de violência obstétrica relatada. A violência obstétrica se destaca em vários pontos desde um atendimento desumanizado a abuso de medicalização e padronização de processos antes naturais para processos invasivos. Retira-se das mulheres a liberdade sobre seus próprios corpos bem como o poder de decidir de qual forma se resultarão os processos reprodutivos. Quando falamos sobre violência obstétrica nota-se um grande reconhecimento nos últimos tempos e vem ganhando espaço em estudos, porém, por outro lado, notamos também que quando se trata de mulher negra pouco é falado, assim, observando a contínua discriminação e racismo da sociedade (Oliveira \& Kubiak, 2019).

Segundo um estudo realizado com 14.625 mulheres brancas e negras de 15 a 49 anos de idade, evidenciou-se a diferença no acesso e na assistência entre mulheres brancas e negras - O mínimo de seis consultas de pré-natal desfavorece as negras, sendo uma média de $78,5 \%$ comparado a $86.2 \%$ das brancas; $39.9 \%$ de parto cesáreos para mulheres negras e $48,8 \%$ paras as brancas; A presença de acompanhantes também é um fato que desfavorece as negras, sendo, 14,3\% para negras e 20,5\% para as brancas (Berquó \& Lago, 2016).

Considerando que a maior vulnerabilidade está dentre as mulheres indígenas, pardas e pretas, tal fato pode-se considerar um alerta para o poder público e para a sociedade. Diante do exposto, evidencia-se a necessidade de um atendimento mais centralizado para esta população de forma a abranger suas necessidades, praticando a equidade e a integralidade, com um maior acompanhamento e de forma mais detalhada no pré-natal, englobando consultas de enfermagem e visitas domiciliares. Com o intuito de reduzir a desigualdade, também torna-se importante a capacitação da equipe, a fim de identificar precocemente as vulnerabilidades, diminuindo a vulnerabilidade programática. Também é fundamental que seja instituído em todos os níveis do sistema de saúde o respeito às leis no 11.108/2005 (Lei do Acompanhante) e nº 11.634/2007 (Lei da Garantia de Vagas), para todas as mulheres, sem distinção de raça/cor (Theophilo et al., 2017).

\section{Políticas Públicas}

O Programa de Humanização no pré-natal e Nascimento (PHPN) foi criado através da portaria/GM n.o 569, de 01/06/2000 pelo Ministério da Saúde, desenvolvido para atender as gestantes nas suas necessidades, o recém-nascido e a mãe que se encontra no período pós-parto (Ministério da Saúde et al., 2002).

Como objetivo primordial, a PHPN visa a facilitação do acesso aos devidos suportes e o acompanhamento ao prénatal, parto e puerpério, na perspectiva dos direitos de cidadania, tendo como fundamento a humanização da assistência para o adequado acompanhamento do parto e puerpério, assim, considerando como prioridades:

"concentrar esforços no sentido de reduzir as altas taxas de morbimortalidade materna, peri e neonatal registradas no país; adotar medidas que assegurem a melhoria do acesso, da cobertura e da qualidade do acompanhamento pré-natal, da assistência ao parto, puerpério e neonatal; ampliar as ações já adotadas pelo Ministério da Saúde na área de atenção à gestante, como os investimentos nas redes estaduais de assistência à gestação de alto risco, o incremento do custeio 
de procedimentos específicos, e outras ações como o Maternidade Segura, o Projeto de Capacitação de Parteiras Tradicionais, além da destinação de recursos para treinamento e capacitação de profissionais diretamente ligados a esta área de atenção, e a realização de investimentos nas unidades hospitalares integrantes destas redes." (Ministério da Saúde, 2002, p. 5).

Em 2003 foi instituída a Política Nacional de Humanização (PNH), tendo como objetivo efetivar os princípios do SUS no cotidiano das práticas de atenção e gestão, qualificando a saúde pública no Brasil e incentivando trocas solidárias entre gestores, trabalhadores e usuários. A PNH deve ser executada e implementada em todas as áreas de atuação do SUS, o que consequentemente pode gerar uma série de melhoras na forma e cuidado e de organização do trabalho (Ministério da Saúde, 2013). Assim sendo, a PNH afirma:

“A humanização é a valorização dos usuários, trabalhadores e gestores no processo de produção de saúde. Valorizar os sujeitos é oportunizar uma maior autonomia, a ampliação da sua capacidade de transformar a realidade em que vivem, através da responsabilidade compartilhada, da criação de vínculos solidários, da participação coletiva nos processos de gestão e de produção de saúde.” (Ministério da Saúde, 2013).

Como parte do processo de humanização, vale ressaltar a importância da validação do sentimento de segurança e conforto proporcionada à gestante através da presença de alguém ao seu lado durante o processo de parto, e para assegurar esse direito, foi instituída a lei do acompanhante (lei federal n 11.108, de 7 de abril de 2005), ao qual especifica os serviços de saúde tanto do SUS quanto de redes privadas próprias ou conveniadas, onde estas têm como obrigação a garantir a gestante o direito à presença de um acompanhante durante o processo de trabalho de parto e pós- parto (Ministério da Saúde et al., 2014).

Com o objetivo de aperfeiçoar ações que dizem respeito a saúde materno-infantil, em 2011 foi instituída a Rede Cegonha, a qual visa a assistência obstétrica e infantil de maior qualidade, adesão às boas práticas de assistência e o desestimulo da adesão desnecessária do parto cesariana (Lago et al., 2020).

Em 2018, a World Health Organization publicou as Recommendations Intrapartum care for a positive childbirth experience, objetivando municiar os profissionais de saúde para as boas práticas no processo do cuidado de parto e pós parto para o binômio mãe-bebê, colocando como prioridade o processo fisiológico. Sendo assim, o Ministério da Saúde vem incentivando a prática do parto normal como uma melhor escolha e conscientizado sobre a importância de mudança na assistência ao parto (Baggio et al., 2021).

Diante do exposto, são compreendidos aspectos fundamentais no que se diz respeito a humanização. Se torna dever da unidade de saúde receber a mulher com dignidade, mas não somente ela como o recém-nascido e seus familiares, trazendo à tona também atitudes éticas e de solidariedade com a parturiente, proporcionando um ambiente mais acolhedor, diferentemente de ambientes que se encontram impostos à mulher nesse período por meio de ações clássicas de unidades de saúde e profissionais que são tradicionalmente desconfortáveis para ela e seus familiares (Ministério da Saúde et al., 2002).

\section{Parto Humanizado: Barreiras para a implementação}

Quando se trata dos serviços de saúde, nota-se que na realidade existe uma fragmentação da assistência, onde o corpo da mulher se torna objeto de intervenções, e a não humanização do processo rompe o cuidado entre a solidariedade, as relações afetivas e a confiança. Muitas mulheres sofrem no padrão obstétrico intervencionista, medicalizado e hegemônico (Silva et al., 2020).

Observa-se que devido ao poder e influência dos médicos, as possibilidades de um novo modelo assistencial são diminuídas, centrando apenas no modelo de assistência intervencionista (Silva et al., 2020).

Segundo a pesquisa Nascer no Brasil, realizada em fevereiro de 2011 a outubro de 2012, evidenciou que o Brasil vivia 
em um cenário de assistências intervencionistas, onde se faziam elevados os riscos para a o binômio mãe-bebê, com o uso de tecnologias desnecessárias e a adoção do parto cirúrgico para mulheres com gravidez de riscos habituais. No ano de 2017, a avaliação da Rede Cegonha evidenciou melhoras e um crescimento na adoção das boas práticas na assistência de trabalhos de partos (Lima et al., 2020).

Destacando a reinserção da Enfermeira Obstétrica (EO) no cenário de assistência ao ciclo gravídico puerperal, observa-se um grande avanço na assistência ao parto no Brasil, tendo em vista que tal atuação prioriza as boas práticas, respeitando a fisiologia e autonomia da mulher, tornando este processo mais humanizado. (Baggio et al., 2021). As instituições que são associadas a atuação de enfermeiras com maior humanização do parto, vêm mudando drasticamente a realidade do modelo vigente da assistência ao parto, que é caracterizado por abusos de tecnologias invasivas, agora, para um parto com maior humanização e satisfação para a mãe (Silva et al., 2020).

A atuação da Enfermagem obstétrica se mostra de grande importância no que tange a diminuição das práticas intervencionistas na assistência ao parto e a adoção do modelo humanizado, uma vez que, a importância de sua atuação nesse sentindo já foi demonstrada em vários estudos e é reconhecida pela Organização Mundial de Saúde (OMS), possibilitando a redução da morbimortalidade perinatal. Tais fatores reforçam a importância da contribuição dessa profissional na prática assistencial, a qual é capaz de reconfigurar o modelo de atenção (Bomfim et al., 2021).

Porém, no que tange aos desafios na realidade de trabalho de alguns profissionais, segundo um estudo realizado com profissionais de enfermagem das unidades de Ginecologia e Obstetrícia e Centro Cirúrgico de hospital universitário do noroeste do Paraná, Brasil, evidencia-se que, fatos como a dinâmica organizacional de trabalho se fazem dificultadoras do processo de humanização do parto destacada pela má administração de tempo e a limitação de funcionários (Ferreira et al., 2019).

Dentre as dificuldades relatadas para a implementação do parto humanizado, foram citadas pelos profissionais que, a ausência de profissionais escalados para o acompanhamento do trabalho de parto é um dos fatores que influenciam negativamente para tal assistência, uma vez que esse déficit limita o fornecimento de orientações essências para a paciente (Ferreira et al., 2019).

Ainda sobre o déficit de profissionais escalados para o acompanhamento do parto, os funcionários da ginecologia e obstetrícia relataram que para a resolução deste problema, se faz necessário a presença de um profissional exclusivo para a garantia de uma assistência humanizada às mulheres em trabalho de parto. Já outros profissionais, ressaltam que a humanização somente ocorrerá de forma efetiva, quando acompanhadas desde o pré-natal (Ferreira et al., 2019).

Outra realidade dificultadora a ser mencionada, é a sobrecarga de trabalho dos profissionais de saúde. Tal fato pode afetar a desenvoltura dos profissionais tanto na assistência aos usuário quanto em sua relação com a equipe. Uma pesquisa sobre a carga de trabalho psíquica em enfermeiros na maternidade do país, evidenciam a dificuldade de conciliação de atividades administrativas e assistenciais, o que influenciam negativamente na qualidade da assistência às parturientes devido à sobrecarga (Ferreira et al., 2019).

Isto posto, o estudo supramencionado também traz à tona a necessidade da reorganização dos recursos humanos, ampliando a demanda de profissionais específicos e capacitados para atenção exclusivas às parturientes (Ferreira et al., 2019).

Já em um estudo realizado em 10 unidades básicas dos municípios de Paracatu, com 10 enfermeiros, evidenciou também como dificuldades para a implementação de uma assistência de parto humanizada, a falta de insumos, problemas com a infraestrutura das unidades, além de grande demanda de gestante para poucos profissionais (Barbosa et al., 2021). Tais fatos mostram a compatibilidade dos problemas entre diversos cenários diferentes.

Em especial na visão dos enfermeiros, nota-se também, a respeito dos obstáculos e barreiras, pouco interesse de alguns profissionais da equipe medica para a pratica dos princípios da humanização. Um grande ponto neste âmbito de 
humanização, pela cultura histórica de institucionalização do parto, a parturiente sofre a mecanização desse evento, melhor dizendo, a redução de um fácil objeto de intervenção, onde se impõe o caminho de conduzir padrões institucionalmente estabelecidos, em que é reconhecido práticas intervencionistas (Monteiro et al., 2020).

\section{Conclusão}

O processo de humanização no parto assume uma postura respeitosa quanto aos desejos e necessidades da mãe e do bebê, levando em conta sempre sua saúde física e mental e seu bem-estar durante o período da gestação, parto e até o puerpério. É importante salientar também, que na humanização é possível que a gestante participe ativamente no momento do nascimento de seu filho, de forma que evite a realização de procedimentos desnecessários, o que gera respostas positivas e benéficas no processo de parturição.

A equipe de enfermagem, por sua vez, realiza o papel de monitoramento da saúde da gestante e do bebê, além de acompanhar também a evolução do parto e suas possíveis complicações, de forma que dê atenção e acolhimento a todos os envolvidos neste processo de maneira digna e humanizada, fazendo-se necessário que os profissionais envolvidos tenham condutas sempre atualizadas e baseadas em estudos científicos a fim de manter boas práticas de assistência.

Diante dos fatos, torna-se imprescindível a adoção de condutas que seguem os direitos legais da mulher dentro do seu cenário de gravidez, levando em consideração o seu direito de escolha e a individualidade de cada uma, apesar das condições de trabalho favorecerem ou não a assistência humanizada.

Dentro deste contexto, a enfermagem deve compreender a sua importância para a experiência que, certamente, marca positivamente ou negativamente a vida de muitas mulheres, a depender principalmente do processo vivido no ambiente de parturição, levando em conta que o seu corpo e a sua vida estarão passando por uma fase de grande importância.

Com o objetivo de aprofundar o conhecimento relacionado aos índices de boas e más práticas no processo de assistência ao parto dentro do cenário hospitalar, sugere-se futuras investigações, seguidas do desenvolvimento de artigos com a temática específica voltada para tal assunto, possibilitando uma melhor delimitação dessas variáveis.

\section{Referências}

Baggio, M. A., Pereira, F. C., Cheffer, M. H., Machineski, G. G., \& Reis, A. C. E. (2021). Significados e experiências de mulheres que vivenciaram o parto humanizado hospitalar assistido por enfermeira obstétrica. Rev. baiana enferm, 35, 42620. 10.18471/rbe.v35.42620.

Barbosa, I. S., Pereira, A. M. M., Costa, N., Dantas, S. L. C., Lima, D. J. M. \& Paiva, A. M. G. (2021). Percepção do enfermeiro da atenção primária acerca do parto humanizado. Enfermagem em Foco, 11(6). 10.21675/2357-707X.2020.v11.n6.3303.

Berquó, E. \& Lago, T. D. G. (2016). Atenção em saúde reprodutiva no Brasil: eventuais diferenciais étnico-raciais. Saúde e Sociedade. 25(3), 550-560. $10.1590 / \mathrm{S} 0104-129020162568$.

Bonfim, A. N. A., Couto, T. M., Lima, K. T. R. S., Almeida, L. T. S., Santos, G. O. \& Santana, A. T. (2021). Percepções de mulheres sobre a assistência de enfermagem durante o parto normal. Rev. baiana enferm, 35, 39087. 10.18471/rbe.v35.39087.

Bourguignon, A. M., \& Grisotti M. (2020). A humanização do parto e nascimento no Brasil nas trajetórias de suas pesquisadoras. História, Ciências, SaúdeManguinhos, 27(2), 485-502. 10.1590/S0104-59702020000200010.

Braide, A. S. G., Brilhante, A. G., Arruda, C. N., Mendonça, F. A. C., Caldas, J. M. P., Nations, M. K., Diógenes, K. C. B. M. \& Amorin, R. F. (2019). Sou homem e pai sim! (Re)construindo a identidade masculina a partir da participação no parto. Revista Panamericana de Salud Pública, 42(190). 10.26633/RPSP.2018.190.

Cordeiro, E. L., Silva, T. M., Silva, L. S. R., Veloso, A. C. F., Pimentel, R. V. T., Cabral, M. M. O., \& Silva, C. M. (2018). A humanização na assistência ao parto e ao nascimento. Revista de Enfermagem UFPE, 12(8), 2154-2162. 10.5205/1981-8963-v12i8a236334p2154-2162-2018.

Editora MS, AMIGO, gravidez, parto e cuidado também são coisas de homem. Seja pai, esteja presente! lei do acompanhante $n^{\circ} 11.108 / 05$ (2014). Folder. Disponível em: https://bvsms.saude.gov.br/bvs/folder/lei_acompanhante.pdf.

Ferreira, M. C., Monteschio, L. V. C., Teston, E. L., Oliveira, L., Serafim, D. \& Marcon, S. S. (2019). Percepções de profissionais de enfermagem sobre humanização do parto em ambiente hospitalar. Rev. Rene, Fortaleza, 20, 41409. 10.15253/2175-6783.20192041409. 
Francisco, B. S., Souza, B. S., Vitório, M. L., Zampieri, M. F. M. \& Gregório, V. R. P. (2015). Percepções dos pais sobre suas vivências como acompanhantes durante o parto e nascimento. Reme: Rev. Min. Enferm. 19(3), 567-575. 10.5935/1415-2762.20150044.

Holanda, S. M., Castro, R. C. M. B., Aquin, P. S., Pinheiro, A. K. B., Lopes, L. G. \& Martins, E. S. (2018). Influência da participação do companheiro no prénatal: satisfação de primíparas quanto ao apoio no parto. Texto \& Contexto - Enfermagem, 27(2). 10.1590/0104-070720180003800016.

Lago, E. L. M., Abrahão, A. L. \& Souza, A. C. (2020). Rede Cegonha, política pública para o cuidado da mulher: revisão integrativa. brazilian journal of nursing, 19(4), 1676-4285. 10.17665/1676-4285.20206437.

Lamy, Z. C., Gonçalves L. L. M., Carvalho, R. H. S. B. F., Alves, M. T. S. B. A., Koser, M. E., Martins, M. S., Leal, N. P. \& Thomaz, E. B. A. F. (2021). Atenção ao parto e nascimento em maternidades do Norte e Nordeste brasileiros: percepção de avaliadores da Rede Cegonha. Ciência \& Saúde Coletiva, 26(3), 951-960. 10.1590/1413-81232021263.26572020.

Leal, M. S., Moreira, R. C. R., Barros, K. C. C., Servo, M. L. S., \& Bispo, T. C. F. (2021) Práticas de humanização no curso do parto na perspectiva de puérperas e enfermeiras obstétricas. Rev. Bras. Enferm, 74, 20190743. 10.1590/0034-7167-2019-0743.

Lima K. L. Raça e violência obstétrica no Brasil. (2016). Monografia (Saúde Coletiva) - Departamento de Saúde Coletiva, Centro de Pesquisas Aggeu Magalhães, Fundação Oswaldo Cruz, 24p. Disponível em: https://www.arca.fiocruz.br/handle/icict/18547.

Lima, M. M., Ribeiro, L. N., Costa, R., Monguilhot, J. J. C., \& Gomes, I. E. M. (2020) Enfermeiras obstétricas no processo de parturição: percepção das mulheres. Rev. Enf. UERJ. 28, 45901. 10.12957/reuerj.2020.45901.

Menezes, M. S. L. \& Comin, F. S. (2019). Envolvimento paterno na relação mãe-bebê: revisão integrativa da literatura. Psicol. rev. 25(1), 19-39. 10.5752/P.1678-9563.2019v25n1p19-39.

Ministério da Saúde. (2002). Programa de humanização do parto: humanização no pré-natal e nascimento. Brasília: Editora MS. Disponível em: https://bvsms.saude.gov.br/bvs/publicacoes/parto.pdf.

Ministério da Saúde. (2002). Programa de Humanização no Pré-natal e Nascimento. Revista Brasileira de Saúde Materno Infantil. 2(1), 69-71. $10.1590 /$ S1519-38292002000100011.

Ministério da Saúde. (2013). Política Nacional de Humanização: Humaniza SUS. Brasília. Disponível em: https://antigo.saude.gov.br/acoes-eprogramas/humanizasus/sobre-o-programa/693-acoes-e-programas/40038-humanizasus.

Monteiro, A. S., Martins E.M., Pereira L.C., Freitas J.C., Silva R. M. \& Jorge H. M. F. (2020). Prática de enfermeiros obstetras na assistência ao parto humanizado em maternidade de alto risco. Rev. Rene, Fortaleza, 21, 43863. 10.15253/2175-6783.20202143863.

Monteiro, M. S. S., Barro, M. J. G., Soares, P. F. B., \& Nunes, R. L. (2020). Importância da assistência de enfermagem no parto humanizado. Revista brasileira interdisciplinar de saúde, 2(4), 51-8. Disponível em: https://revistarebis.rebis.com.br/index.php/rebis/article/view/139.

Moura, J. W. S., Leite, J. C. S., Oliveira, V. R. \& Silva, J. P. X. (2020). Humanização do parto na perspectiva da equipe de enfermagem de um Centro de Parto Normal. Enfermagem em Foco. 11(3). 10.21675/2357-707X.2020.v11.n3.3256.

Oliveira, B. M. C. \& Kubiak, F. R. (2019). Racismo institucional e a saúde da mulher negra: uma análise da produção científica brasileira. Saúde em Debate, 43(122), 939-948. 10.1590/0103-1104201912222.

Quitete, J. B. \& Monteiro, J. A. M. B. (2018). A participação do pai no parto domiciliar planejado: um ato significativo para a mulher. Revista Enfermagem UERJ, 26, 18682. 10.12957/reuerj.2018.18682.

Ribeiro, J. F., Sousa, Y. E., Luz, V. L. E. S., Coelho, D. M. M., Feitosa, V. C., Calvalcante, M. F. A., Barbosa, A. K. C. \& Silva, T. C. A. (2018). Percepção do pai sobre a sua presença durante o processo parturitivo. Revista de Enfermagem UFPE. 12(6), 1586-1592. 10.5205/1981-8963-v12i6a234522p1586-15922018.

Russo, J. A., \& Nucci, M. F. (2020). Parindo no paraíso: parto humanizado, ocitocina e a produção corporal de uma nova maternidade. Interface Comunicação, Saúde, Educação, 24, 180390. 10.1590/Interface.180390.

Silva, G. F., Moura, M. A. V., Queiroz, A. B. Z., Pereira, A. L. F., Carvalho A. L. O. \& Netto, L. A. (2020). Possibilidades para a mudança do modelo obstétrico hegemônico pelas enfermeiras obstétricas. Revista Enfermagem UERJ, 28, 49421. 10.12957/reuerj.2020.49421.

Theophilo, R. L., Rattner, D. \& Pereira, E. L. (2018). Vulnerabilidade de mulheres negras na atenção ao pré-natal e ao parto no SUS: análise da pesquisa da Ouvidoria Ativa. Ciência \& Saúde Coletiva. 23(11), 3505-3516. 10.1590/1413-812320182311.31552016.

Villamil, M. M. L., Botero, M. P. A. \& Guzmán, C. I. C. (2020). Atención humanizada del embarazo: la mirada de gestantes que acuden a una unidad hospitalaria de salud. Enfermería Actual de Costa Rica, (38), 180-195. 10.15517/revenf.v0i38.38376. 